\title{
ANALYSIS OF STOCHASTIC QUANTIZATION FOR THE FRACTIONAL EDWARDS MEASURE
}

\author{
WOLFGANG BOCK AND TORBEN FATTLER
}

\begin{abstract}
In [6] the existence of a diffusion process whose invariant measure is the fractional polymer or Edwards measure for fractional Brownian motion in dimension $d \in \mathbb{N}$ with Hurst parameter $H \in(0,1)$ fulfilling $d H<1$ is shown. The diffusion is constructed via Dirichlet form techniques in infinite dimensional (Gaussian) analysis. By providing a Fukushima decomposition for the stochastic quantization of the fractional Edwards measure we prove that the constructed process solves weakly a stochastic differential equation in infinite dimension for quasi-all starting points. Moreover, the solution process is driven by an Ornstein-Uhlenbeck process taking values in an infinite dimensional distribution space and is unique, in the sense that the underlying Dirichlet form is Markov unique. The equilibrium measure, which is by construction the fractional Edwards measure, is specified to be an extremal Gibbs state and therefore, the constructed stochastic dynamics is time ergodic. The studied stochastic differential equation provides in the language of polymer physics the dynamics of the bonds, i.e. stochastically spoken the noise of the process. An integration leads then to polymer paths. We show that if one starts with a continuous polymer configuration the integrated process stays almost surely continuous during the time evolution.
\end{abstract}

\section{INTRODUCTION}

For a given probability measure $\nu$ on a measurable space $(X, \mathcal{F})$ the stochastic quantization of $\nu$ means the construction of a Markov process which has $\nu$ as an invariant measure. Stochastic quantization has been studied first by Parisi and $\mathrm{Wu}$ for applications in quantum field theory, which were extended to Euclidean quantum fields, see [33. The Markov process, which is obtained, is parametrized w.r.t. to a new time parameter, which is often denoted as 'compute time'. This notion is due to the fact, that one can use the stochastic quantization in order to construct a numerical scheme to sample path with a given probability distribution, [12].

The two-dimensional polymer measure or Edward's measure is informally given as

$$
d \mu_{g}=Z^{-1} e^{-g L} d \mu_{0},
$$

where $\mu_{0}$ denotes the Wiener measure, $L$ the self-intersection local time of Brownian motion and $Z$ is a normalization constant. The self-intersection local time can be interpreted as the time the process spends on its trajectory - or in particular - it counts the self-crossings the process undertakes. The Edwards measure thus penalizes every selfintersection exponentially. Note however that it gives for $g<\infty$ only weakly self-avoiding paths, i.e. the path is allowed to cross itself but with a growing number of self-intersections the next self-intersection is becoming more and more unlikely.

Albeverio, Röckner, Hu and Zhou use Dirichlet form methods to construct a Markov process associated to the Edwards measure in two dimensions [2].

Also intersection local times $L$ of Brownian motion have been studied for a long time and by many authors, see e.g. [2], 4], 8], 9], [13], 18], 22], 24] and [35]- 40], the intersections of Brownian motion paths have been studied even since the Forties, see e.g. 23]. One can consider intersections of sample paths with themselves or e.g. with other, independent Brownian motions e.g. [39, one can study simple 9] or $n$-fold intersections e.g. [10, 24] and one can ask all of these questions for linear, planar, spatial or - in general - $d$-dimensional Brownian motion: selfintersections become increasingly scarce as the dimension $d$ increases. A well-written monograph about self-avoiding random walks is provided by N. Madras and G. Slade [25].

A somewhat informal but very suggestive definition of self-intersection local time of a Gaussian process $Y$ is in terms of an integral over Dirac's - or Donsker's - $\delta$-function

$$
L(Y) \equiv \int d^{2} t \delta\left(Y\left(t_{2}\right)-Y\left(t_{1}\right)\right)
$$

where for now $Y=B$ is a Brownian motion, intended to sum up the contributions from each pair of "times" $t_{1}, t_{2}$ for which the process $Y$ is at the same point, see e.g. [7. In Edwards' modeling of long polymer molecules by Brownian motion paths, $L$ is used to model the "excluded volume" effect: different parts of the molecule should not

Date: September 26, 2018. 
be located at the same point in space. As another application, Symanzik 35 introduced $L$ as a tool in constructive quantum field theory.

A rigorous definition, such as e.g. through a sequence of Gaussians approximating the $\delta$-function, will lead to increasingly singular objects and will necessitate various "renormalizations" as the dimension $d$ increases. For $d>1$ the expectation will diverge in the limit and must be subtracted, see e.g. [22, [36, as a side effect such a local time will then no more be positive. For $d>3$ various further renormalizations have been proposed in [37. that will make $L$ into a well-defined generalized function of Brownian motion. For $d=3$ a multiplicative renormalization gives rise to an independent Brownian motion as the weak limit of regularized and subtracted approximations to $L$, see [40; another renormalization has been constructed by Westwater to make the Gibbs factor $\exp (-g L)$ of the polymer model well-defined, see 38 .

In this article we first introduce the setting along the lines of White Noise or Gaussian Analysis, using the fractional White Noise measure. This can be compared to the approach in [29. Moreover the results from [32] concerning the gradient are extended to this setting. In [6] the stochastic quantization for the fractional Edwards measure $\nu_{g}$ is studied using the framework of Dirichlet forms. The existence of a Markov process which has $\nu_{g}$ as invariant measure is based on the results of [16] and [17, which show that the self-intersection local time in the case $H d<1$ is Meyer-Watanabe differentiable. The closability of the gradient Dirichlet form is then shown by an integration by parts argument. The irreducibility follows as in the Brownian case, see [2].

In this article we carryout a further analysis of the underlying objects. Using the theory of Dirichlet forms we show that the stochastic quantization of the fractional Edwards measure solves weakly a stochastic differential equation (SDE) in infinite dimension given by

$$
d X_{t}=\sqrt{2} d W_{t}+b\left(X_{t}\right) d t, \quad X_{0}=\omega,
$$

for quasi-all starting points $\omega$ in an infinite dimensional state space $\mathcal{N}^{\prime}$. Here the drift term $b$ is determined by the gradient of the self-intersection local time of fractional Brownian motion with Hurst parameter $H$ fulfilling $H d<1$, where $d \in \mathbb{N}$ denotes space dimension. The solution process is driven by a Brownian motion having an intrinsic linear drift. Such a process is due to 21] characterized as an Ornstein-Uhlenbeck process taking values in an infinite dimensional space. The solution process is unique, in the sense that the underlying Dirichlet form is Markov unique. The equilibrium measure, which is by construction the fractional Edwards measure, is specified to be the extremal Gibbs state. The SDE under consideration provides in the language of polymer physics the dynamics of the bonds, i.e. stochastically spoken the noise of the process. An integration leads then to polymer paths. We show that if one starts with a continuous polymer configuration during the evolution the integrated process stays almost surely continuous.

\section{FrameWORK}

For $d \in \mathbb{N}$ and Hurst parameter $H \in(0,1)$ a fractional Brownian motion in dimension $d$ is a $\mathbb{R}^{d}$-valued centered Gaussian process $\left(B_{t}^{H}\right)_{t \geq 0}$ with covariance

$$
\operatorname{cov}_{H}(t, s):=\mathbb{E}\left[B_{t}^{H} B_{s}^{H}\right]=\frac{1}{2}\left(t^{2 H}+s^{2 H}-|t-s|^{2 H}\right), \quad s, t \in[0, \infty),
$$

defined on a probability space $(\Omega, \mathcal{F}, P)$. Here $\mathbb{E}$ denotes the mathematical expectation with respect to the probability measure $P$. For $s \in(0, \infty)$ let $\Theta_{s}:=\mathbb{1}_{[0, s)}$ and set $\left(\Theta_{s}, \Theta_{t}\right)_{H}:=\operatorname{cov}_{H}(t, s)$ for $s, t \in[0, \infty)$. Moreover, let $X:=\operatorname{span}\left\{\Theta_{s} \mid s>0\right\}$. Hence $x, y \in X$ are simple functions of the form

$$
x=\sum_{i=1}^{n} \alpha_{i} \Theta_{s_{i}}, \quad y=\sum_{j=1}^{m} \beta_{j} \Theta_{t_{j}},
$$

with $n, m \in \mathbb{N}$ and

$$
(x, y)_{H}:=\sum_{i=1}^{n} \sum_{j=1}^{m} \alpha_{i} \beta_{j}\left(\Theta_{s_{i}}, \Theta_{t_{j}}\right)_{H},
$$

defines an inner product on $X$. Taking the abstract completion of the inner product space $\left(X,(\cdot, \cdot)_{H}\right)$ we obtain a Hilbert space $\left(\mathcal{H},(\cdot, \cdot)_{H}\right)$, where the scalar product extending $(\cdot, \cdot)_{H}$ to $\mathcal{H}$ is denoted by the same symbol.

Moreover, $\left(\mathcal{H},(\cdot, \cdot)_{H}\right)$ has a countable orthonormal basis $\beta=\left(\eta_{k}\right)_{k \in \mathbb{N}}$. For $k \in \mathbb{N}$ let $\lambda_{k} \in \mathbb{R}$ such that

$$
1<\lambda_{1}<\lambda_{2}<\ldots<\lambda_{k}<\lambda_{k+1}<\ldots \quad \text { and } \quad \sum_{k=1}^{\infty} \frac{1}{\lambda_{k}^{2}}<\infty .
$$


Next we consider

$$
\mathcal{H} \ni f \mapsto A f:=\sum_{k=1}^{\infty} \lambda_{k}\left(f, \eta_{k}\right)_{H} \eta_{k} \in \mathcal{H}
$$

and define for $p \in \mathbb{N}$

$$
\mathcal{H}_{p}:=\left\{f \in \mathcal{H} \mid\left\|A^{p} f\right\|_{H}<\infty\right\} \quad \text { and } \quad \mathcal{N}:=\bigcap_{p \in \mathbb{N}} \mathcal{H}_{p},
$$

where $\|\cdot\|_{H}$ denotes the induced norm on $\mathcal{H}$. Then $\mathcal{N}$ is a countably Hilbert space, which is Fréchet and nuclear, compare e.g. 30. Its topological dual is given by

$$
\mathcal{N}^{\prime}:=\bigcup_{p \in \mathbb{N}} \mathcal{H}_{-p}
$$

for an analogous construction see e.g. 15]. Thus we obtain the Gel'fand triple

$$
\mathcal{N} \subset \mathcal{H} \subset \mathcal{N}^{\prime}
$$

In what follows we denote complexifications by a subscript $\mathbb{C}$.

Now by the Bochner-Minlos-Sazonov theorem, see e.g. [5] or [14, we define a Gaussian measure $\mu_{H}$ on $\mathcal{N}^{\prime}$ by

$$
\int_{\mathcal{N}^{\prime}} \exp \left(i\langle\omega, \xi\rangle_{H}\right) d \mu_{H}(\omega):=\exp \left(-\frac{1}{2}\|\xi\|_{H}\right) \text {. }
$$

Remark 2.1. Note that the measure has full support, i.e. every open set has positive measure. This can be seen by [20, Theorem 6] or the fact that the measure is quasi translation invariant w.r.t. shifts in direction of the subspace $\mathcal{N}$ which is dense in $\mathcal{N}^{\prime}$, compare e.g. [15, Chapter 4B].

We obtain the probability space $\left(\mathcal{N}^{\prime}, \mathcal{C}_{\sigma}, \mu_{H}\right)$. Here $\mathcal{C}_{\sigma}\left(\mathcal{N}^{\prime}\right):=\sigma\left(\mathcal{C}_{F_{1}, \ldots, F_{n}}^{\xi_{1}, \ldots, \xi_{n}}\right)$ denotes the $\sigma$-algebra of cylinder sets

$$
\mathcal{C}_{F_{1}, \ldots, F_{n}}^{\xi_{1}, \ldots, \xi_{n}}=\left\{\omega \in \mathcal{N}^{\prime} \mid\left\langle\xi_{1}, \omega\right\rangle_{H} \in F_{1}, \ldots,\left\langle\xi_{n}, \omega\right\rangle_{H} \in F_{n}, \xi_{i} \in \mathcal{N}, F_{j} \in \mathcal{B}(\mathbb{R}), j=1, \ldots, n, n \in \mathbb{N}\right\},
$$

where $\mathcal{B}(\mathbb{R})$ denotes the $\sigma$-algebra of Borel sets in $\mathbb{R}$.

Note that since $\mathcal{N}$ is a nuclear countably Hilbert space we have, see e.g. [15]:

$$
\mathcal{C}_{\sigma}\left(\mathcal{N}^{\prime}\right)=\mathcal{B}_{w}\left(\mathcal{N}^{\prime}\right)=\mathcal{B}_{s}\left(\mathcal{N}^{\prime}\right)
$$

where $\mathcal{B}_{w}\left(\mathcal{N}^{\prime}\right)$ (resp. $\left.\mathcal{B}_{s}\left(\mathcal{N}^{\prime}\right)\right)$ is the Borel $\sigma$-algebra generated by the weak (resp. strong) topology.

We define by

$$
\mathcal{P}:=\left\{p \in L^{2}\left(\mathcal{N}^{\prime} ; \mu_{H}\right) \mid p(\omega)=\sum_{n=0}^{N}\left\langle\omega^{\otimes n}, f^{\otimes n}\right\rangle_{H}, \quad f \in \mathcal{N}_{\mathbb{C}}\right\}
$$

the space of smooth polynomials.

In 6 the authors construct the stochastic quantization of the fractional Edwards measure via a local Dirichlet form. Here we briefly sketch the construction and summarize facts from the differential calculus, needed in this framework.

Definition 2.2. Let $p \in \mathcal{P}$ and $\left(\eta_{k}\right)_{k \in \mathbb{N}} \subset \mathcal{N}$ a CONS of $\mathcal{H}$. Setting

$$
\left(D_{\eta_{k}} p\right)(\omega)=\lim _{\lambda \rightarrow 0} \frac{p\left(\omega+\lambda \eta_{k}\right)-p(\omega)}{\lambda}=\sum_{n=1}^{N} n\left\langle\eta_{k} \otimes \omega^{\otimes n-1}, f^{\otimes n}\right\rangle_{H}, \quad \omega \in \mathcal{N}^{\prime}
$$

we define

$$
\nabla p:=\left(D_{\eta_{k}} p\right)_{k=1}^{\infty} .
$$

Remark 2.3. Note that this defines $D_{\eta_{k}}$ and $\nabla$ on a dense subspace of $L^{2}\left(\mathcal{N}^{\prime} ; \mu_{H}\right)$.

For $p \in \mathcal{P}$ we have

$$
\begin{aligned}
\sum_{k=1}^{\infty}\left(D_{\eta_{k}} p\right)^{2}(\omega)=\sum_{k=1}^{\infty} \sum_{m=1}^{N} \sum_{n=1}^{N} m n\left\langle\omega^{\otimes m-1} \otimes \eta_{k}, f^{\otimes m}\right\rangle_{H}\left\langle\omega^{\otimes n-1} \otimes \eta_{k}, f^{\otimes n}\right\rangle_{H} & \\
& =\sum_{m=1}^{N} m \sum_{n=1}^{N} n\left\langle\omega^{\otimes m+n+2},(f, f)_{H} f^{\otimes n+m-2}\right\rangle_{H} .
\end{aligned}
$$


Remark 2.4. Furthermore for $u \in \mathcal{H}$ the adjoint $D_{u}^{*}=\langle\cdot, u\rangle_{H}-D_{u}$ on a dense subspace, e.g. polynomials in $L^{2}\left(\mathcal{N}^{\prime} ; \mu_{H}\right)$, see e.g. [32].

In the following we will just write $L$ for $L\left(B^{H}\right)$, the self-intersection local time of $B^{H}, H \in\left(0, \frac{1}{d}\right)$, where $B^{H}$ is a $d$-dimensional fractional Brownian motion with Hurst parameter $H$.

Definition 2.5. By $\nu_{g}:=\exp (-g L) \mu_{H}$ we denote the fractional Edwards measure. Moreover, $L^{2}\left(\mathcal{N}^{\prime} ; \nu_{g}\right)$ is the corresponding space of square integrable functions equipped with the inner product $(\cdot, \cdot)_{L^{2}\left(\mathcal{N}^{\prime} ; \nu_{g}\right)}$.

Remark 2.6. Note that since $\exp (-g L) \in L^{2}\left(\mathcal{N}^{\prime} ; \mu_{H}\right)$ with $d H<1$, we have in this case that $\nu_{g}$ is absolutely continuous w.r.t. $\mu_{H}$ for all $g>0$, see e.g. [16].

Theorem 2.7. The bilinear form

$$
\mathcal{E}_{\nu_{g}}(u, v):=\mathbb{E}_{H}(\exp (-g L) \nabla u \cdot \nabla v), \quad u, v \in \mathcal{P},
$$

is a densely defined, closable, symmetric pre-Dirichlet form and gives rise to a local, quasi-regular Dirichlet form $\left(\mathcal{E}_{\nu_{g}}, D\left(\mathcal{E}_{\nu_{g}}\right)\right)$ in $L^{2}\left(\mathcal{N}^{\prime} ; \mu_{H}\right)$. Here $\mathbb{E}_{H}$ denotes expectation w.r.t. $\mu_{H}$.

Proof. See [6, Theorem 3.1].

Remark 2.8. (i) In particular, Remark 2.1provides that the bilinear form in Theorem 2.7]is well-defined. More precisely, the full support of the measure insures that the gradient respects the $\mu_{H}$-classes (hence also the $\nu_{g}$-classes) determined by $\mathcal{P}$.

(ii) Due to Theorem 2.7 we have that

$$
\mathcal{E}_{\nu_{g}}(u, v)=\sum_{k=1}^{\infty}\left(D_{\eta_{k}} u, D_{\eta_{k}} v\right)_{L^{2}\left(\mathcal{N}^{\prime} ; \nu_{g}\right)}, \quad u, v \in \mathcal{P},
$$

is a densely defined, closable, symmetric classical gradient pre-Dirichlet form and gives rise to a local, quasi-regular Dirichlet form $\left(\mathcal{E}_{\nu_{g}}, D\left(\mathcal{E}_{\nu_{g}}\right)\right)$ in $L^{2}\left(\mathcal{N}^{\prime} ; \nu_{g}\right)$.

(iii) Moreover, since $1 \in \mathcal{P}$ and $\mathcal{E}_{\nu_{g}}(1,1)=0$, due to [11, Theo. 1.6.3], the local, quasi-regular Dirichlet form $\left(\mathcal{E}_{\nu_{g}}, D\left(\mathcal{E}_{\nu_{g}}\right)\right)$ in $L^{2}\left(\mathcal{N}^{\prime} ; \nu_{g}\right)$ is recurrent.

(iv) Due to [27, Chapter II, Section 3 d)] (i) implies that $(\nabla, \mathcal{P})$ is closable in $L^{2}\left(\mathcal{N}^{\prime} ; \nu_{g}\right)$. We denote the closure of $\nabla$ by the same symbol.

(v) As in [15, Corollar 10.8] we obtain that the closures of $\left(\mathcal{E}_{\nu_{g}}, \mathcal{P}\right)$ and $\left(\mathcal{E}_{\nu_{g}}, \mathcal{F} C_{b}^{\infty}\right)$ coincide. Here and below $\psi \in \mathcal{F} C_{b}^{\infty}$ is of the form

$$
\psi(\omega)=f\left(\left\langle\xi_{1}, \omega\right\rangle_{H}, \ldots,\left\langle\xi_{n}, \omega\right\rangle_{H}\right), \quad f \in C_{b}^{\infty}\left(\mathbb{R}^{n}\right), \xi_{j} \in \mathcal{N}, j \in\{1, \ldots, n\}, n \in \mathbb{N}, \omega \in \mathcal{N}^{\prime} .
$$

By Friedrichs representation theorem we have the existence of the self-adjoint generator $\left(A_{\nu_{g}}, D\left(A_{\nu_{g}}\right)\right)$ corresponding to $\left(\mathcal{E}_{\nu_{g}}, D\left(\mathcal{E}_{\nu_{g}}\right)\right)$.

Proposition 2.9. There exists a unique, positive, self-adjoint, linear operator $\left(A_{\nu_{g}}, D\left(A_{\nu_{g}}\right)\right)$ on $L^{2}\left(\mathcal{N}^{\prime} ; \nu_{g}\right)$ such that

$$
D\left(A_{\nu_{g}}\right) \subset D\left(\mathcal{E}_{\nu_{g}}\right) \quad \text { and } \quad \mathcal{E}_{\nu_{g}}(u, v)=\left(A_{\nu_{g}} u, v\right)_{L^{2}\left(\mathcal{N}^{\prime} ; \nu_{g}\right)} \quad \text { for all } u \in D\left(A_{\nu_{g}}\right), v \in D\left(\mathcal{E}_{\nu_{g}}\right) .
$$

Proof. Using Remark 2.8 this is a direct application of [11, Coro. 1.3.1].

For functions $u \in \mathcal{P}$ the next result provides a nice representation of the operator from the above proposition.

Proposition 2.10. For $u \in \mathcal{P}$ the generator $A_{\nu_{g}}$ in Proposition 2.9 has the form

$$
A_{\nu_{g}} u=-\mathcal{L} u:=N u-g \nabla u \cdot \nabla L,
$$

where $N u:=\sum_{k=1}^{\infty} D_{\eta_{k}}^{*} D_{\eta_{k}} u$ is the so-called number operator. 
Proof. For $u, v \in \mathcal{P}$ we have

$$
\begin{aligned}
& \mathcal{E}_{\nu_{g}}(u, v)=\sum_{k=1}^{\infty} \int_{\mathcal{N}^{\prime}} D_{\eta_{k}} u D_{\eta_{k}} v e^{-g L} d \mu_{H}=\sum_{k=1}^{\infty} \int_{\mathcal{N}^{\prime}}\left(D_{\eta_{k}} u e^{-g L}\right) D_{\eta_{k}} v d \mu_{H} \\
&= \sum_{k=1}^{\infty} \int_{\mathcal{N}^{\prime}} D_{\eta_{k}}^{*}\left(\left(D_{\eta_{k}} u\right) e^{-g L}\right) v d \mu_{H}=\sum_{k=1}^{\infty} \int_{\mathcal{N}^{\prime}}\left(\left\langle\cdot, \eta_{k}\right\rangle_{H} D_{\eta_{k}} u e^{-g L}-D_{\eta_{k}}\left(D_{\eta_{k}} u e^{-g L}\right)\right) v d \mu_{H} \\
&= \sum_{k=1}^{\infty} \int_{\mathcal{N}^{\prime}}\left(\left\langle\cdot, \eta_{k}\right\rangle_{H} D_{\eta_{k}} u e^{-g L}-\left(D_{\eta_{k}} D_{\eta_{k}} u e^{-g L}-\left(D_{\eta_{k}} u\right) g\left(D_{\eta_{k}} L\right) e^{-g L}\right)\right) v d \mu_{H} \\
&= \sum_{k=1}^{\infty} \int_{\mathcal{N}^{\prime}}\left(\left\langle\cdot, \eta_{k}\right\rangle_{H} D_{\eta_{k}} u-D_{\eta_{k}} D_{\eta_{k}} u+D_{\eta_{k}} u g D_{\eta_{k}} L\right) v e^{-g L} d \mu_{H} \\
&=\sum_{k=1}^{\infty} \int_{\mathcal{N}^{\prime}}\left(D_{\eta_{k}}^{*} D_{\eta_{k}} u+g\left(D_{\eta_{k}} u\right)\left(D_{\eta_{k}} L\right)\right) v e^{-g L} d \mu_{H} .
\end{aligned}
$$

Thus by using the so-called number operator

$$
N u=\sum_{k=1}^{\infty} D_{\eta_{k}}^{*} D_{\eta_{k}} u
$$

for $u \in \mathcal{P}$ we obtain

$$
\mathcal{E}_{\nu_{g}}(u, v)=(-\mathcal{L} u, v)_{L^{2}\left(\mathcal{N}^{\prime} ; \nu_{g}\right)}=(N u-g \nabla u \cdot \nabla L, v)_{L^{2}\left(\mathcal{N}^{\prime} ; \nu_{g}\right)}, \quad u, v \in \mathcal{P} .
$$

Hence for $u \in \mathcal{P}$ the generator $\mathcal{L}$ is given by

$$
\mathcal{L} u:=-N u-g \nabla u \cdot \nabla L .
$$

Proposition 2.11. The generator $\left(A_{\nu_{g}}, D\left(A_{\nu_{g}}\right)\right.$ ) in Proposition 2.9 is the only Dirichlet operator extending $\left(-\mathcal{L}, \mathcal{F} C_{b}^{\infty}\right)$, where $-\mathcal{L} u=N u-g \nabla u \cdot \nabla L$ for $u \in \mathcal{F} C_{b}^{\infty}$, see Proposition $[$.10.

Proof. Due to Remark 2.8(iv) we have that $A_{\nu_{g}} u=-\mathcal{L} u=N u-g \nabla u \cdot \nabla L$ for all $u \in \mathcal{F} C_{b}^{\infty} \cdot \operatorname{Since} \exp (-g L)$ and $\nabla \exp (-g L)$ are square-integrable w.r.t. $\mu_{H}$, see [16], and $\mu_{H}$ is Gaussian, we obtain the statement by using [34, Theorem 2.3].

Remark 2.12. The property provided in Proposition 2.11 is known as Markov uniqueness.

Let $\left(T_{t}^{\nu_{g}}\right)_{t \geq 0}$ with $T_{t}^{\nu_{g}}:=\exp \left(-t A_{\nu_{g}}\right), t \geq 0$, denote the corresponding strongly continuous contraction semigroup on $L^{2}\left(\mathcal{N}^{\prime} ; \nu_{g}\right)$, cf. e.g. [27, Chap. I, Sect. 1,2].

Remark 2.13. $\left(T_{t}^{\nu_{g}}\right)_{t \geq 0}$ is recurrent due to Remark 2.8(iii). Using [11, Lemma 1.6.5] we obtain that $\left(T_{t}^{\nu_{g}}\right)_{t \geq 0}$ is conservative.

Abstract Dirichlet form theory provides the following results, compare e.g. [11] or [27]:

Theorem 2.14. There exists a diffusion process $\mathbf{M}=\left(\boldsymbol{\Omega}, \mathcal{F},\left(\mathcal{F}_{t}\right)_{t \geq 0},\left(X_{t}\right)_{t \geq 0},\left(\mathbf{P}_{\omega}\right)_{\omega \in \mathcal{N}^{\prime}}\right)$ with state space $\mathcal{N}^{\prime}$ which is properly associated with $\left(\mathcal{E}_{\nu_{g}}, D\left(\mathcal{E}_{\nu_{g}}\right)\right)$, i.e., for all $\left(\nu_{g}\right.$-versions of $) f \in L^{2}\left(\mathcal{N}^{\prime}, \nu_{g}\right)$ and all $t \geq 0$ the function

$$
\mathcal{N}^{\prime} \ni \omega \mapsto\left(p_{t} f\right)(\omega):=\int_{\Omega} f\left(X_{t}\right) d \mathbf{P}_{\omega} \in \mathbb{R},
$$

is an $\mathcal{E}_{\nu_{g}}$-quasi-continuous version of $T_{t}^{\nu_{g}} f . \mathbf{M}$ is up to $\nu_{g}$-equivalence unique (cf. [27, Chap. IV, Sect. 6]). In particular, $\mathbf{M}$ is $\nu_{g}$-symmetric, i.e.,

$$
\int_{\mathcal{N}^{\prime}}\left(p_{t} f\right) g d \nu_{g}=\int_{\mathcal{N}^{\prime}} f\left(p_{t} g\right) d \nu_{g}
$$

for all bounded measurable functions $f, g: \mathcal{N}^{\prime} \rightarrow \mathbb{R}, t>0$, as well as conservative, i.e., $p_{t} \mathbb{1}=\mathbb{1} \mathcal{E}_{\nu_{g}}-q . e$. for all $t \geq 0$ or in other words the diffusion process $\mathbf{M}$ is of infinite life time. Thus $\nu_{g}$ is an invariant measure for $\mathbf{M}$. 
Theorem 2.15. The diffusion process $\mathbf{M}$ as given in Theorem 2.14 is solving the martingale problem for $\left(A_{\nu_{g}}, D\left(A_{\nu_{g}}\right)\right)$, i.e., for all $u \in D\left(A_{\nu_{g}}\right)$,

$$
u\left(X_{t}\right)-u\left(X_{0}\right)-\int_{0}^{t}\left(A_{\nu_{g}} u\right)\left(X_{s}\right) d s, \quad t \geq 0,
$$

is an $\left(\mathcal{F}_{t}\right)_{t \geq 0}$-martingale under $\mathbf{P}_{\omega}$ (hence starting in $\omega$ ) for $\mathcal{E}_{\nu_{g}}$-quasi all $\omega \in \mathcal{N}^{\prime}$.

Proof. The statement follows by Theorem 2.7 and [3, Theorem 3.4(i)].

\section{IRREDUCiBILITy AND EXTREMAL GiBBs STATES}

In this section we provide important consequences of irreducibility of the considered bilinear form. In particular, this means invariance of the associated diffusion process under time translations. For the stochastic quantization of the fractional Edwards measure, this has been shown in [6] and relates to a particular class of measures.

Definition 3.1. For $K \subset \mathcal{H}, \mathcal{B}\left(\mathcal{N}^{\prime}\right)$-measurable functions $b_{k}, k \in K$, and $b:=\left(b_{k}\right)_{k \in K}$ we define $\mathcal{G}^{b}$ to be the set of all probability measures $\mu$ on $\mathcal{B}\left(\mathcal{N}^{\prime}\right)$ such that for all $k \in K, b_{k} \in L^{2}\left(\mathcal{N}^{\prime} ; \nu_{g}\right)$ and the following integration by parts formula holds:

$$
\int_{\mathcal{N}^{\prime}} \frac{\partial u}{\partial k} d \mu=-\int_{\mathcal{N}^{\prime}} u b_{k} d \mu \text { for all } u \in \mathcal{P}
$$

where $\frac{\partial u}{\partial k}(\omega):=\left.\frac{d}{d s} u(\omega+s k)\right|_{s=0}, \omega \in \mathcal{N}^{\prime}$. Elements in $\mathcal{G}^{b}$ are called Gibbs states associated with $b$.

Remark 3.2. Definition 3.1 coincides with the Definition of a Gibbs state in the sense of [3] due to Remark 2.8(iii) and (iv).

Using Remark 2.4 we obtain

Lemma 3.3. For $g \geq 0$ the measures $\widetilde{\nu_{g}}:=\frac{1}{Z} \nu_{g}$, where $Z \in(0, \infty)$ is a normalizing constant, are contained in $\mathcal{G}^{b}$ with $b=\left(b_{k}\right)_{k \in K}$, where $b_{k}:=\langle k, \cdot\rangle_{H}+\langle k, g \nabla L\rangle_{H}$ and $K:=\operatorname{span} \beta$.

Remark 3.4. Note that $Z$ is the expectation of $\exp (-g L)$ with respect to $\mu_{H}$, which exists due to [16].

In [6] the following theorem is shown.

Theorem 3.5. There exists a constant $c_{0}>0$, such that for all $g<c_{0}$ the form $\left(\mathcal{E}_{\nu_{g}}, D\left(\mathcal{E}_{\nu_{g}}\right)\right)$ is irreducible $($ i.e. $u \in D\left(\mathcal{E}_{\nu_{g}}\right)$ with $\left(\mathcal{E}_{\nu_{g}}(u, u)=0\right.$ implies $u$ is a constant), equivalently the associated diffusion is invariant under time translations.

This has immediate consequences for the diffusion process $\mathbf{M}=\left(\boldsymbol{\Omega}, \mathcal{F},\left(\mathcal{F}_{t}\right)_{t \geq 0},\left(X_{t}\right)_{t \geq 0},\left(\mathbf{P}_{\omega}\right)_{\omega \in \mathcal{N}^{\prime}}\right)$ given in Theorem 2.14

Corollary 3.6. There exists a constant $c_{0}>0$, such that for all $g<c_{0}$ we have that

$$
\lim _{t \rightarrow \infty} \frac{1}{t} \int_{0}^{t} f\left(X_{t}\right) d s=\int_{\mathcal{N}^{\prime}} f d \widetilde{\nu_{g}}
$$

$\mathbf{P}_{\omega}$-almost surely for quasi every $\omega \in \mathcal{N}^{\prime}$ and all $f \in L^{1}\left(\mathcal{N}^{\prime} ; \widetilde{\nu_{g}}\right)$.

Proof. Due to [11, Theo. 4.7.3(iii)] it is sufficient to show that $\left(\mathcal{E}_{\nu_{g}}, D\left(\mathcal{E}_{\nu_{g}}\right)\right)$ is irreducible recurrent. Recurrence follows by [11, Theo. 1.6.3], since $1 \in \mathcal{P}$ and $\mathcal{E}_{\nu_{g}}(u, u)=0$. Irreducibility is provided by Theorem 3.5 .

Remark 3.7. The property obtained in Corollary 3.6 is know as time ergodicity of the process $\left(X_{t}\right)_{t \geq 0}$.

Next we introduce extremal Gibbs states.

Definition 3.8. A measure $\mu \in \mathcal{G}^{b}$ is called extremal if it can not be written as convex combination of elements in the set $\mathcal{G}^{b}$. This we denote by $\mu \in \mathcal{G}_{\text {ext }}^{b}$.

Definition 3.9. Let $\nu$ be a measure on $\left(\mathcal{N}^{\prime}, \mathcal{B}\left(\mathcal{N}^{\prime}\right)\right.$. A $\mathcal{B}\left(\mathcal{N}^{\prime}\right)$-measurable (real valued) function $f$ is called $K$-shift invariant if $f(\omega+t k)=f(\omega)$ for $\nu$-a.e. $\omega \in \mathcal{N}^{\prime}$, for all $t \in \mathbb{R}$ and all $k \in K . g \in L^{2}\left(\mathcal{N}^{\prime} ; \nu\right)$ is called $K$-shift invariant if there exists a $\mathcal{B}\left(\mathcal{N}^{\prime}\right)$-measurable representative which is $K$-shift invariant.

Corollary 3.10. In situation of Theorem 3.5 we have that

(i) $\widetilde{\nu_{g}} \in \mathcal{G}_{\text {ext }}^{b}$, i.e., $\widetilde{\nu_{g}}$ is an extremal Gibbs state.

(ii) $\widetilde{\nu_{g}}$ is $K$-ergodic, i.e., every $K$-invariant $\mathcal{B}(H)$-measurable function is $\widetilde{\nu_{g}}$-a.e. constant.

(iii) the semigroup $\left(T_{t}^{\nu_{g}}\right)_{t \geq 0}$ is irreducible. 
Proof. Apply [1, Theorems 1.2, 3.7 and Propositopn 2.3].

Remark 3.11. (i) Using a standard approximation argument via the CONS of $\mathcal{H}$ we even obtain the results of Corollary 3.10 for $K=\mathcal{H}$.

(ii) The property obtained in Corollary 3.10(ii) is known as shift ergodicity of the process $\left(X_{t}\right)_{t \geq 0}$.

\section{Characterization of the underlying stochastic Differential EQuation}

Abstract Dirichlet form theory provides the following statement, see e.g. 3, Theo. 4.3].

Theorem 4.1 (Fukushima decomposition). Let $u \in D\left(\mathcal{E}_{\nu_{g}}\right)$ and $\widetilde{u}$ a quasi-continuous $\nu_{g}$-version of $u$. Then the additive functional $\left(\widetilde{u}\left(X_{t}\right)-\widetilde{u}\left(X_{0}\right)\right)_{t \geq 0}$ of $\mathbf{M}$ can be uniquely represented as

$$
\widetilde{u}\left(X_{t}\right)-\widetilde{u}\left(X_{0}\right)=M_{t}^{[u]}+N_{t}^{[u]}, \quad t \geq 0,
$$

where $M^{[u]}:=\left(M_{t}^{[u]}\right)_{t \geq 0}$ is a MAF (martingale additive functional) of $\mathbf{M}$ of finite energy and $N^{[u]}:=\left(N_{t}^{[u]}\right)_{t \geq 0}$ is a CAF (continuous additive functional) of $\mathbf{M}$ of zero energy. Recall that $\mathbf{M}$ is provided in Theorem 2.14.

For $k \in K=\operatorname{span} \beta$ and $\omega \in \mathcal{N}^{\prime}$ we define $u_{k}(\omega):=\langle k, \omega\rangle_{H}$.

Remark 4.2. In the situation of Theorem 4.1, we have for $k \in K$ that $u_{k} \in D\left(A_{\nu_{g}}\right)$ and $A_{\nu_{g}} u_{k}=b_{k}$. This immediately implies that for $k \in K, N^{\left[u_{k}\right]}=\left(N_{t}^{\left[u_{k}\right]}\right)_{t \geq 0}$ in (4.1) reads

$$
\left.\left(N_{t}^{\left[u_{k}\right]}\right)_{t \geq 0}=\left(\int_{0}^{t} b_{k}\left(X_{s}\right)\right) d s\right)_{t \geq 0}
$$

by Theorem 2.15. Moreover, $\left(M_{t}^{\left[u_{k}\right]}, \mathcal{F}_{t}, \mathbf{P}_{\omega}\right)_{t \geq 0}$ is a martingale which is hence also continuous with $M_{0}^{\left[u_{k}\right]}=0$. Since

$$
2 \mathcal{E}_{\nu_{g}}\left(u_{k} \cdot \mathbb{1}, u_{k}\right)-\mathcal{E}_{\nu_{g}}\left(u_{k}^{2}, \mathbb{1}\right)=2\|k\|_{H}^{2},
$$

the quadratic variation $\left\langle M^{\left[u_{k}\right]}\right\rangle_{t}$ for $t \geq 0$ is given by

$$
\left\langle M^{\left[u_{k}\right]}\right\rangle_{t}=2 t\|k\|_{H}^{2}
$$

If $\|k\|_{H}=1$ it follows by P. Levy's characterization of Brownian motion and its scaling properties that $\left(M_{t}^{\left[u_{k}\right]}\right)_{t \geq 0}$ is an $\left(\mathcal{F}_{t}\right)_{t \geq 0}$-Brownian motion $\left(W_{t}^{k}\right)_{t>0}$ scaled by $\sqrt{2}$ starting at zero under each $\mathbf{P}_{\omega}$ for $\omega \in \mathcal{N}^{\prime} \backslash S_{k}$, where $S_{k} \subset \mathcal{N}^{\prime}$ is a set with capacity zero. This Brownian motion is associated to a gradient bilinear form w.r.t. the reference measure $\mu_{H}$. The associated generator is given by the number operator $N$, see (2.3). Due to [21] such a process defines an Ornstein-Uhlenbeck process. In this sense, the appearing Brownian motion $\left(W_{t}^{k}\right)_{t \geq 0}$ has an intrinsic linear drift.

Corollary 4.3. Let $k \in K$. Then the decomposition (4.1) reads

$$
u_{k}\left(X_{t}\right)-u_{k}\left(X_{0}\right)=\sqrt{2} W_{t}^{k}+\int_{0}^{t} b_{k}\left(X_{t}\right) d s, \quad t \geq 0,
$$

where for all $\omega \in \mathcal{N}^{\prime} \backslash S_{k}$ for some $S_{k} \subset \mathcal{N}^{\prime}$ with capacity zero, the continuous martingale $\left(M_{t}^{\left[u_{k}\right]}, \mathcal{F}_{t}, P_{z}\right)_{t \geq 0}$ is a Brownian motion scaled by $\sqrt{2}$ and $b_{k}=\langle k, \cdot\rangle_{H}+\langle k, g \nabla L\rangle_{H}$ is as in Lemma 3.3.

Lemma 4.4. Let $k, k^{\prime} \in K$. Then

$$
\left\langle W^{k}, W^{k^{\prime}}\right\rangle_{t}=t\left(k, k^{\prime}\right)_{H}
$$

under $P_{\omega}$ (as given in Theorem 2.14) for quasi-every $\omega \in \mathcal{N}^{\prime}$, where $\left(W_{t}^{k}\right)_{t \geq 0}$ is as in (4.2).

Proof. See [3, Lemm. 5.4]

This immediately implies the next proposition, see [3, Prop. 5.5].

Proposition 4.5. For $d \in \mathbb{N}$ let $\eta_{1}, \ldots, \eta_{d} \in \beta$. Then $\bar{W}_{t}:=\left(W_{t}^{k_{1}}, \ldots W_{t}^{k_{d}}\right), t \geq 0$, is a d-dimensional $\left(\mathcal{F}_{t}\right)_{t \geq 0}$ Brownian motion starting at zero under each $P_{\omega}$ for $\omega \in \mathcal{N}^{\prime} \backslash S_{k}$, where $S_{k} \subset \mathcal{N}^{\prime}$ is a set with capacity zero. 
Moreover, we have by [3, Theo. 5.7] the following theorem.

Theorem 4.6. For quasi every $\omega \in \mathcal{N}^{\prime},\left(\left\{\langle k, \omega\rangle_{H} \mid k \in \beta\right\}, \mathcal{F}_{t}, P_{\omega}\right)_{t \geq 0}$ solves the following system of stochastic differential equations

$$
\begin{gathered}
d Y_{t}=\sqrt{2} d W_{t}^{k}+b_{k}\left(\left(Y_{t}^{k}\right)_{k} \in \beta\right) d t \\
Y_{0}^{k}=\langle k, z\rangle_{H}
\end{gathered}, k \in \beta,
$$

where $\left\{\left(W_{t}^{k}\right)_{t \geq 0} \mid k \in \beta\right\}$ is a collection of independent one dimensional $\left(\mathcal{F}_{t}\right)_{t \geq 0}$-Brownian motions starting at zero, where we identify $z \in \mathcal{N}^{\prime}$ with $\left(\langle k, z\rangle_{H}\right)_{k \in \beta}$, and $b_{k}=\langle k, \cdot\rangle_{H}+\langle k, g \nabla L\rangle_{H}$ is as in Lemma 3.3.

Remark 4.7. Theorem4.6just says that using the corresponding Dirichlet form we have constructed a weak solution of (4.3), which is unique by Proposition 2.11 and Theorem 2.15.

Applying [3, Theo. 6.10] we obtain the following main result.

Theorem 4.8. For $\mathbf{M}=\left(\boldsymbol{\Omega}, \mathcal{F},\left(\mathcal{F}_{t}\right)_{t \geq 0},\left(X_{t}\right)_{t \geq 0},\left(\mathbf{P}_{\omega}\right)_{\omega \in \mathcal{N}^{\prime}}\right)$, see Theorem 2.14, there exists a map W: $\mathcal{N}^{\prime} \rightarrow$ $C\left([0, \infty), \mathcal{N}^{\prime}\right)$ such that for quasi all $\omega \in \mathcal{N}^{\prime}$ under $\mathbf{P}_{\omega}, W=\left(W_{t}\right)_{t \geq 0}$ is an $\left(\mathcal{F}_{t}\right)_{t \geq 0}$-Brownian motion on $\mathcal{N}^{\prime}$ starting in zero with covariance $(\cdot, \cdot)_{H}$ such that for quasi every $\omega \in \mathcal{N}^{\prime}$ we have the unique representation

$$
X_{t}=\omega+\sqrt{2} W_{t}+\int_{0}^{t} b\left(X_{s}\right) d s, \quad t \geq 0, \quad \mathbf{P}_{\omega}-a . s .
$$

with $b=\left(b_{k}\right)_{k \in \beta}$, where $b_{k}=\langle k, \cdot\rangle_{H}+\langle k, g \nabla L\rangle_{H}$.

Remark 4.9. Theorem 4.8 just says that the diffusion process $\mathbf{M}$ in Theorem 2.14 provides a unique weak solution to the stochastic differential equation

$$
d X_{t}=\sqrt{2} d W_{t}+b\left(X_{t}\right) d t, \quad X_{0}=\omega,
$$

with $b=\left(b_{k}\right)_{k \in \beta}$, where $b_{k}=\langle k, \cdot\rangle_{H}+\langle k, g \nabla L\rangle_{H}$.

\section{Continuity OF POLYMer PATHS}

The stochastic differential equation we obtained by the Fukushima decomposition gives in every compute time point $t$ a noise for a path, which for large compute times, due to the long time behavior of the process is distributed according to the law of a weakly self-avoiding fractional Brownian motion starting in 0. However, since we constructed the process by stochastic quantization on the level of the noise, the question is, if we have polymer paths which are continuous.

Here we want to emphasize that we are not talking about the continuous paths properties of the process which is given by the SDE (4.4), but more about the continuity property of the process at a compute time point $t$ integrated out on the function time interval $[0, \tau]$. The property thus is also dependent on the construction of the space.

Proposition 5.1. For all $H \in(0,1)$ the stochastic process $\left\langle\cdot, \mathbb{1}_{[0, \tau)}\right\rangle_{H}$ has $\mu_{H}$-almost surely continuous paths. In other words: For almost every $\omega \in \mathcal{N}^{\prime}$ the mapping $\tau \mapsto\left\langle\omega, \mathbb{1}_{[0, \tau)}\right\rangle$ is continuous.

A proof via Kolmogorov-Chentsov can be found e.g. in [26, 31].

Remark 5.2. Note that the SDE (4.3) provides in the language of polymer physics the dynamics of the bonds, i.e. stochastically spoken the noise of the process. An integration leads then to polymer paths. Here this is done by a dual pairing with the indicator functions, which exists in the sense of an $L^{2}\left(\mathcal{N}^{\prime} ; \mu_{H}\right)$-limit, compare [15].

Proposition 5.3. For an initial state $\omega \in \mathcal{N}^{\prime}$ with $\tau \mapsto\left\langle\omega, \mathbb{1}_{[0, \tau)}\right\rangle$ is continuous the Markov process $\mathbf{M}$ given by Theorem 4.8 fulfills for every compute time point $t$ that

$$
t \mapsto\left\langle X_{t}(\omega), \mathbb{1}_{[0, \tau)}\right\rangle
$$

is $\mu_{H}$-almost surely continuous.

Proof. By Proposition 5.1 we have that $\left\langle\cdot, \mathbb{1}_{[0, t)}\right\rangle_{H}$ has $\mu_{H}$-almost surely continuous paths. Denote by $R_{t}$ the set of points in $\mathcal{N}^{\prime}$ which can be reached by $X$ at time point $t$ with $X_{0}=\omega$. Therefore it suffices to show, that $\mu_{H}\left(R_{t}\right)>0$ for all $t$. Since an Ornstein-Uhlenbeck process puts mass on such a non-null set for every compute time point $t$, see e.g [21] the assertion is shown.

Remark 5.4. The above proposition makes sure that if one starts with a continuous polymer configuration during the evolution the process stays almost surely continuous. 
Acknowledgement: We truly thank L. Streit for helpful discussions. Financial support by the mathematics department of the University of Kaiserslautern for research visits at Lisbon are gratefully acknowledged.

\section{REFERENCES}

[1] Albeverio, S., Kondratiev, Yu., Röckner, M.: Ergodicity of $L^{2}$-Semigroups and Extremality of Gibbs States. Journal of Func. Ana.. 144, 394-423. (1997).

[2] Albeverio, S., Hu,Y.-Z.,Röckner, M., Zhou, X. Y.: Stochastic Quantization of the Two-Dimensional Polymer Measure. Appl. Math. Optim. 40, 341-354. (1996).

[3] Albeverio, S., Röckner: Dirichlet form methods for uniqueness of martingale problems and applications. in Stochastic Analysis, Ithaca, NY, 1993, Proceedings of Symposia in Pure Mathematics, Volume 57, 513-528. (AMS, Providence, RI, 1995).

[4] Bass, R. F., Khoshnevisan, D., Intersection Local Times and Tanaka Formulas. Ann. Inst. H. Poincaré, $29,(1993), 419-451$.

[5] Berezansky, Yu. M., Kondratiev, Yu. G., Spectral Methods in Infinite-Dimensional Analysis. Naukova Dumka, Kiev, 1988 (in Russian). English translation, Kluwer Academic Publishers, Dordrecht, 1995.

[6] Bock, W. and Fattler T. and Streit L., Stochastic Quantization for the fractional Edwards Measure. accepted for publication in Acta Applicandae Mathematicae.

[7] de Faria, M., Hida, T., Streit, L., Watanabe, H., Intersection local times as generalized white noise functionals. Acta Appl. Math., 46:351-362, 1997.

[8] de Faria, M., Drumond, C., Streit, L., The renormalization of self-intersection local times. I. The chaos expansion. Infin. Dimens. Anal. Quantum Probab. Relat. Top., 3(2):223-236, 2000.

[9] Dvoretzky, A., Erdös, P., Kakutani, S.Double points of paths of Brownian motion in n-space. Acta Sci. math. Szeged, 12, (1950), 75-81.

[10] Dvoretzky, A., Erdös, P., Kakutani, S., Taylor, S. J., Triple points of the Brownian motion in 3-space. Proc. Cambridge Philos. Soc. 53 (1957), 856-862.

[11] Fukushima, M., Oshima, Y., Takeda, M.: Dirichlet Forms and Symmetric Markov Processes. de Gruyter, Berlin, (1994)

[12] Guha, A. and Lee, S.-C., Stochastic quantization for numerical simulation. Physics Letters B, 134(3):216-220, (1984).

[13] He, S. W., Yang, W. Q., Yao, R. Q., Wang, J. G., Local times of self-intersection for multidimensional Brownian motion. Nagoya Math. J. 138 (1995), 51-67.

[14] Hida, T., Stationary Stochastic Processes, Mathematical Notes Series, Princeton University Press, Princeton 1970.

[15] Hida, T., Kuo, H.H., Potthoff, J. and Streit, L., White Noise. An infinite dimensional calculus. Kluwer, Dordrecht, 516p., (1993)

[16] Hu, Y.: Self-intersection local time of fractional Brownian motions - via chaos expansion. J. Math. Kyoto Univ. 41, 233-250 (2001).

[17] Hu, Y., Nualart, D., Song, J., Integral representation of renormalized self-intersection local times. J. Func. Ana., Vol 255, No. 9 , (2008)

[18] Imkeller, P., Pérez-Abreu, V., Vives, J., Chaos expansions of double intersection local times of Brownian motion in $\mathbb{R}^{d}$ and renormalization. Stochastic Process. Appl. 56 (1995), 1-34.

[19] Kato, T., Perturbation theory for linear operators, 2nd edition, SpringerVerlag, Berlin-Heidelberg-New York, (1976).

[20] Kondratiev, Y.G., Streit, L. and Westerkamp, W., A note on positive distributions in Gaussian analysis, Ukr. Mat. J., Vol. 47, No. $5,(1996)$

[21] Kuo H.H., White noise distribution theory, Kluwer Academic Publishers, Dordrecht, 1993.CRC Press, Boca Raton, 1996

[22] Le Gall, J. F.: Le Gall, J. F., Sur le temps local d'intersection du mouvement brownien plan et la méthode de renormalisation de Varadhan. Sém. Prob. XIX, Lecture Notes in Mathematics 1123, Springer, Berlin, 1985, 314-331.

[23] Lévy, P.: Lévy, P., Le mouvement brownien plan. Amer. J. Math. 62 (1940), 487-550.

[24] Lyons, T. J., The critical dimension at which quasi-every Brownian motion is self-avoiding. Adv. in Appl. Probab. (Spec. Suppl. 1986), 87-99.

[25] Madras, N. and Slade, G., The self-avoiding walk. Modern Birkhäuser Classics, (2013).

[26] Y. Mishura, Stochastic Calculus for Fractional Brownian Motion and Related Processes. Springer LNM 1929 (2008).

[27] Ma, Z.-M. and Röckner, M., An introduction to the theory of (non-symmetric) Dirichlet forms, Springer, Berlin, (1992).

[28] Nualart, D., The Malliavin calculus and related topics., Second edition. Probability and its Applications (New York). SpringerVerlag, Berlin, (2006).

[29] Hu, Y. and Øksendal, B.,Fractional White Noise Calculus and Applications to Finance. Infin. Dimens. Anal. Quantum. Probab. Relat. Top. Vol. 06, Nr. 1 (2003).

[30] Obata N., White Noise Calculus and Fock Spaces, volume 1577 of LNM, Springer Verlag, Berlin, Heidelberg, New York, 1994.

[31] F. Biagini, Y. Hu, B. Oksendal, Stochastic Calculus for Fractional Brownian Motion and Applications. Springer, Berlin, 2007.

[32] Potthoff, J.: On differential operators in White Noise analysis. Acta Applicandae Mathematicae 63, 333-347 (2000).

[33] Parisi, G., Wu Y.-S.: Perturbation theory without gauge fixing. Sci. Sinica 24, (1981).

[34] Röckner, M., Zhang, T.-Sh.: Uniqueness of Generalized Schrödinger Operators and Applications. Journal of Functional Analysis 105, 187-203 (1994).

[35] Symanzik, K., Euclidean quantum field theory, in "Local Quantum Theory" (R. Jost, ed.), Academic Press, New York, 1969.

[36] Varadhan, S. R. S., Appendix to "Euclidean quantum field theory" by K. Symanzik, in "Local Quantum Theory" (R. Jost. ed.), Academic Press, New York, 1969.

[37] Watanabe, H., The local time of self-intersections of Brownian motions as generalized Brownian functionals. Lett. Math. Phys. 23 (1991), 1-9.

[38] Westwater, J., On Edward's model for long polymer chains. Comm. Math. Phys. 72 (1980), 131-174.

[39] Wolpert, R., Wiener path intersection and local time. J. Funct. Anal. 30 (1978), 329-340.

[40] Yor, M., Renormalisation et convergence en loi pour les temps locaux d'intersection du mouvement brownien dans $\mathbb{R}^{3}$. Séminaire de Probabilité, Lecture Notes in Mathematics 1123, Springer, Berlin, 1985, 350-365. 
Technomathematics Group, University of Kaiserslautern

E-mail address: bock@mathematik.uni-kl.de

Functional Analysis and Stochastic Analysis Group, University of Kaiserslautern

E-mail address: fattler@mathematik.uni-kl.de 